\title{
A Novel Angiographic Index Can Independently Predict 1-Year Cardiovascular Outcomes after Anterior ST-Elevation Myocardial Infarction
}

\author{
ISMAIL M. IBRAHIM, M.D.; MOHAMED TAHA ELSAID, M.Sc.; MAHMOUD ABDELAZIZ, M.D.; \\ MOHAMED I. ALAWADI, M.D. and AHMED EL ZAYAT, M.D.
}

The Department of Cardiology, Faculty of Medicine, Zagazig University, Zagazig, Egypt

\begin{abstract}
Background: In ST-elevation myocardial infarction (STEMI), the association between either the grade or location of coronary thrombi with major adverse cardiovascular events (MACE) is controversial.

Aim of Study: We created a novel index "thrombus score" through multiplying the TIMI thrombus grade by a segment weighing factor adopted from Gensini score. We hypothesized this thrombus score can better reflect the prognostic relevance of coronary thrombi.

Patients and Methods: We prospectively studied 364 consecutive patients with anterior STEMI treated by primary percutaneous coronary intervention (PCI). Patients were followed-up for major adverse cardiovascular events (MACE) defined as the composite of repeated myocardial infarction, target vessel revascularization, stent thrombosis, stroke; and all-cause mortality.

Results: 47 (12.9\%) patients developed MACE. In the MACE group, percentage of female patients $(p=0.004)$, baseline and peak HsTnT $(<0.001$; each), thrombus score $(p<0.001)$ were significantly higher. Conversely, LVEF at discharge was significantly lower in the MACE group $(p=0.02)$. Using Cox regression analysis, only thrombus score $(p<0.001)$ and LVEF $(p=0.01)$ were found as independent predictors of MACE. By ROC curve analysis, thrombus score at a cutoff value of $>$ ! 7.5 was associated with MACE at 1 year with a sensitivity of $85 \%$ and specificity of $90 \%$ (AUC: $0.87,95 \%$ CI: $0.83-0.93$, $p<0.001)$.

Conclusion: In STEMI patients treated with primary PCI, the novel angiographic "thrombus score" was the strongest independent predictor of MACE during the first year. Further studies are recommended to better understand and validate this novel score.
\end{abstract}

Key Words: Myocardial infarction - Primary percutaneous coronary intervention - Major adverse cardiovascular events - MACE - Thrombus score.

Correspondence to: Dr. Ismail M. Ibrahim, E-Mail: Dr heartimi@yahoo.com

\section{Introduction}

APPLYING mechanical reperfusion therapy for ST-elevation myocardial infarction (STEMI) has tremendously improved immediate outcome [1] However, in-hospital and long-term outcomes are still poor [2,3]. One of major determinants of clinical outcomes associated with STEMI is the final infarct size [4]. The latter is determined by the extent of myocardium at risk, state of collaterals, and myocardial preconditioning [5]. Intuitively, extent of myocardium at risk depends on the magnitude of reduction of myocardial perfusion, and the length of the ischemic time. Reduction of myocardial perfusion depends on, among other factors, degree of coronary occlusion (i.e. plaque volume and TIMI thrombus grade) and anatomical location of the culprit lesion (proximal versus mid versus distal).

Before extensive use of glycoprotein IIb/IIIa inhibitors, thrombus burden was found to be associated with short and long-term major adverse cardiovascular events (MACE) [6-8]. However, recent studies failed to show an association between thrombus burden and MACE after STEMI $[9,10]$ Likewise, the association between proximity of culprit lesion location and MACE after STEMI was debatable among studies [11-13]. Therefore, we postulated that combining both parameters in one index might better reflect the prognostic relevance of coronary thrombi.

The new angiographic score is calculated through multiplyingTIMI thrombus grade by a segment weighing factor (adopted from modified Gensini score [14]) (Fig. 1). In our proposed "thrombus score", a thrombus of TIMI grade 2, for example, corresponds to thrombus score of 5 and 1 in 
proximal and distal left anterior descending (LAD) artery, respectively.

\section{Patients and Methods}

1-Study population: We conducted a prospective, non-randomized studyincluded 364 consecutive patients with their first anterior STEMI involving LAD artery referred for primary percutaneous coronary intervention (PCI) in Zagazig University Hospital during the period from July 2018 till July 2020. Diagnostic criteria for anterior STEMI were typical chest pain lasting for more than 30 minutes in association with: (1) ST segment elevation at least $1 \mathrm{~mm}(2 \mathrm{~mm}$ for V1-V3) in 2 or more contiguous precordial leads or new onset of left bundlebranch block; and (2) Increasedhigh sensitive Troponin T (HsTnT) [15] . Patients with nonthrombus containing culprits as well as those with cardiogenic shock were excluded to avoid the bias of outliers (patients with expected low and high mortality; respectively).

2- Clinical examination and laboratory measurements: Hypertension was defined as prior use of an antihypertensive drug or a systolic blood pressure $>140$ and/or a diastolic blood pressure $>90$ during index hospitalization [16]. Diabetes mellitus (DM) was defined as use of hypoglycemic drugsor $\mathrm{HbA} 1 \mathrm{C}>6.5 \mathrm{~g} / \mathrm{dL}$ during index hospitalization [17]. Hypercholesterolemia was defined as prior use of statins or fasting total cholesterol more than $200 \mathrm{mg} / \mathrm{dL}$. Positive family history of premature CAD was defined as presence of CAD in a close relative (women $<65$ and men $<55$ years). Smoking during the last month was considered current smoking. Drug history was taken from each patient. Additionally, admission blood pressure, heart rate, Killipclass, pain to balloon time and door to balloon time were recorded for each patient. Surface electrocardiogram was recorded for each patient at admission and at 90 minutes after primary PCI to calculate percentage of ST segment resolution. Baseline and peak HsTnT, baseline creatinine as well as HbA1c were measured. Modification of Diet in Renal Disease formula was used to calculate estimated glomerular filtration rate (eGFR) [18] .

3- Primary percutaneous coronary intervention $(P C I)$ : All patients underwent primary PCI through femoral approach by two operators who were blinded to the study design. Before the procedure, patients were given a loading dose of clopidogrel $(300 \mathrm{mg})$ and aspirin $(300 \mathrm{mg})$. The use of aspiration catheters and balloon pre-dilatation were left to the discretion of the operators. In case of no-reflow, intracoronary (IC) tirofiban was given as a bailout therapy.

Initial thrombolysis in myocardial infarction (TIMI) thrombus grade was assessed as previously described [19]. After crossing the lesion with a guide-wire and/or small balloon dilatation, TIMI thrombus grade 5 was re-stratified according to the previous scale [15]. The final TIMI thrombus grade was used in our score. Additionally, myocardial blush grade (MBG), final TIMI flow as well as corrected TIMI frame count (cTFC) were assessed as previously described [20-22]

Calculation of thrombus score: The final TIMI thrombus grade was multiplied by a culprit segment weighing factor derived from modified Gensini score [14] (Fig. 1). TIMI grades of thrombi located in proximal segment of LAD (i.e. before the first major septal) were multiplied by 2.5 . TIMI grades for those located in mid segment of LAD (i.e. between the first major septal and extending to the point where LAD forms an angle in RAO view) were multiplied by 1.5. TIMI grades for those located in distal segment of LAD (i.e. distal to LAD angle and extending to or beyond the apex) were multiplied by 1 . In case of thrombi located in two contiguous segments, the higher factor was used. The score was calculated by two independent investigators blinded to patients' history and outcomes.

4- Clinical outcomes: Patients were prescribed the maximum tolerated doses of drugs recommended by the latest guidelines on STEMI [15]. None of patients failed to comply with the prescribed medications. MACE was defined as the composite of repeat myocardial infarction, target vessel revascularization (TVR), stentthrombosis, stroke; and all-cause mortality. Thestudy protocol was approved by the local ethicscommittee (ZU-IRB \#4723/37-2018). A written informed consent was obtained from each patient.

5- Statistical analysis: Continuous data were presented as mean $\pm \mathrm{SD}$ andwere compared using the Student's $t$-test (in case of normality) or MannWhitney U test (in case of non-normality). Categorical data were presented as frequencies (percentages) and were compared using the Chi-square or Fisher exact test. The effects of different variables on MACE were analyzed using Cox proportional regression. Correlation between thrombus score and both CTFC and MBG (as indirect indicators of myocardial microvascular function) was performed. The best cutoff value of thrombus score associated with MACE at 1 year was determined 
using receiver operating characteristic (ROC) curve analysis. A $p$-value of less than 0.05 was considered significant. All tests were performed using SPSS version 20 (SPSS Inc., Chicago, IL).

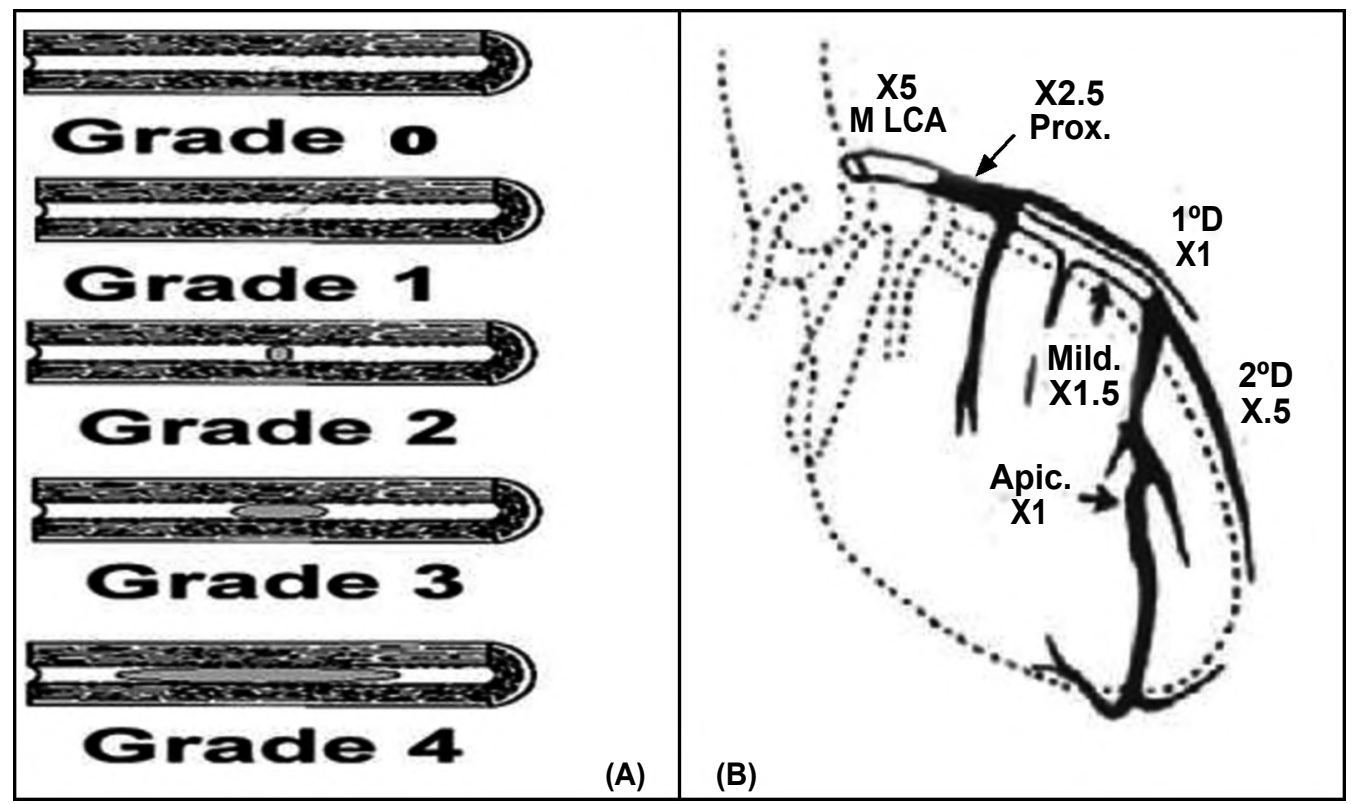

Fig. (1): Method of calculation of the new angiographic "thrombus score" through multiplying TIMI thrombus grade* as shown in (A) by a culprit segment weighing factor** as shown in (B).

* TIMI thrombus grade is assessed as classified by Gibson et al. [15]

** Segment weighing factor is adopted from Gensini score [14].

\section{Results}

1- MACE development: Of the 364 anterior STEMI patients managed with timely primary PCI, $47(12.9 \%)$ patients developed MACE. Median follow-up period (including the in-hospital period of the index STEMI) was 11 months (interquartile range: 10-14 months). During follow-up; repeat myocardial infarction developed in 3 patients $(0.8 \%)$, TVR in 16 patients (4.4\%), stentthrombosis in 2 patients $(0.5 \%)$, stroke in 7 patients $(1.9 \%)$, and all-cause mortality in 31 patients $(8.5 \%)$. Patients were classified into MACE group $(n=47$ patients) and no MACE group (n-317 patients).

2- Comparison between baseline characteristics of both groups: Comparison between baseline clinical and laboratory findings of both groups is shown in Table (1). Percentage of female gender was significantly higher in the MACE group $(p=0.004)$. Similarly, baseline and peak HsTnT were significantly higher in MACE the group $(p<0.001$; each). On the contrary, prevalence of $>50 \%$ ST resolution at 90 minutes was significantly lower in the MACE group ( $p=0.03)$. Similarly, baseline eGFR and left ventricular ejection fraction (LVEF) at discharge was significantly lower in the MACE group ( $p=0.04$ and 0.02 ; respectively).

Comparison between baseline procedural findings of both groups is shown in Table (2). Longer pain to balloon time ( $p=0.01$ ), higher TIMI thrombus grade $(p=0.02)$, higher percentage of proximal location of culprit lesion $(p=0.001)$ and thrombus score $(p<0.001)$ were observed in the MACE group. The prevalnce of ICtirofiban administration was significantly higher in the MACE group $(p=0.01)$. Post-procedural TIMI flow 2-3 and MBG 2-3 were observed less frequently in the MACE group $(p<0.001$; each). Significantly higher cTFC was found in the MACE group $(p<0.001)$. The prevalnce of no-reflow was higher among patients in the MACE group $(p<0.001)$.

3- Cox regression analysis for prediction of MACE: Only thrombus score $(p<0.001)$ and LVEF at discharge $(p=0.01)$ were found as independent predictors of MACE. On the contrary, neither TIMI thrombus grade nor proximal lesion location could independently predict MACE ( $p=0.30$ and 0.16 ; respectively) (Table 3 ).

4- Correlation between thrombus score and $c T F C$ and $M B G$ : Performing Pearson's correlation analysis, significant positive correlation was found between thrombus score and cTFC ( $r=0.26$, $p<0.001)$. On the other hand, using Spearman's correlation analysis, significant negative correlation was found between thrombus score and MBG $(r=-0.31, p<0.001)$ (Fig. 2). 
5- ROC curve analysis to determine the best cutoff value of thrombus score associated with $M A C E$ at 1 year: Thrombus score at a cutoff value of $\geq 7.5$ was associated with MACE at 1 year with a sensitivity of $85 \%$ and specificity of $90 \%$ (area under curve: $0.87,95 \%$ CI: $0.83-0.93, p<0.001$ ) (Fig. 3).

6- Intra- and inter-observer agreement:Two blinded interventional cardiologists (IMI and AE) evaluated the coronary angiographies of 80 randomly selected patients in order to estimate of inter-observer and intra-observer agreements for thrombus scorecalculation. Inter-observer variability calculated using Bland-Altman test was nonsignificant $(p=0.56$ ). After 2 weeks, thrombus score was re-calculated by one of the 2 interventional cardiologists (IMI) and inter-observer variability was also non-significant ( $p=0.93$ ).

Table (1): Comparison between clinical characteristics of both groups.

\begin{tabular}{|c|c|c|c|c|}
\hline & $\begin{array}{c}\text { Total } \\
(n=364)\end{array}$ & $\begin{array}{l}\text { No MACE } \\
\quad(n=317)\end{array}$ & $\begin{array}{l}\text { MACE } \\
(n=47)\end{array}$ & $\begin{array}{c}p- \\
\text { value }\end{array}$ \\
\hline Age (years) & $56.1 \pm 7.2$ & $57.5 \pm 6.3$ & $55.7 \pm 5.8$ & 0.082 \\
\hline Female gender, $\mathrm{n}(\%)$ & $146(40.1 \%)$ & $116(36.6 \%)$ & $30(63.8 \%)$ & 0.004 \\
\hline Hypertension, n (\%) & $246(67.6 \%)$ & $217(68.5 \%)$ & $29(61.7 \%)$ & 0.49 \\
\hline Diabetes mellitus, n (\%) & $183(50.3 \%)$ & $158(49.8 \%)$ & $25(53.2 \%)$ & 0.30 \\
\hline Dyslipidemia, n (\%) & $176(48.4 \%)$ & $149(47.0 \%)$ & $27(57.0 \%)$ & 0.27 \\
\hline Current smoking, n (\%) & $238(65.4 \%)$ & $206(65.0 \%)$ & $32(68.1 \%)$ & 0.08 \\
\hline Family history of premature CAD & $57(15.7 \%)$ & $48(15.1 \%)$ & $9(19.1 \%)$ & 0.38 \\
\hline CHA2DS2-VASC score & $2.98 \pm 1.16$ & $2.92 \pm 0.96$ & $3.22 \pm 0.76$ & 0.06 \\
\hline ACEI/ARB use before index STEMI, n (\%) & $138(37.9 \%)$ & $122(38.5 \%)$ & $16(32.9 \%)$ & 0.31 \\
\hline Beta-blockers use before index STEMI, n (\%) & $193(53.0 \%)$ & $170(53.6 \%)$ & $23(48.9 \%)$ & 0.62 \\
\hline Admission heart rate, beat/minute & $79.7(11.4)$ & $78.1(10.4)$ & $83.2(16.2)$ & 0.10 \\
\hline Admission systolic blood pressure, $\mathrm{mmHg}$ & $147.4(18.1)$ & $157.0(24.8)$ & $142.4(30.2)$ & 0.12 \\
\hline In-hospital Killip class $>1, \mathrm{n}(\%)$ & $68(18.7 \%)$ & $53(16.7 \%)$ & $15(31.9 \%)$ & 0.07 \\
\hline Baseline HsTnT (ng/L) & $738.4 \pm 541.5$ & $601.1 \pm 474.1$ & $837.6 \pm 394.1$ & $<0.001$ \\
\hline Peak HsTnT (ng/L) & $22890.4 \pm 13447.4$ & $15023.8 \pm 11763.4$ & $29925.1 \pm 13372.2$ & $<0.001$ \\
\hline $\mathrm{HbA} 1 \mathrm{c}(\mathrm{g} / \mathrm{dl})$ & $7.4(1.7)$ & $6.4(1.8)$ & $7.5(1.9)$ & 0.13 \\
\hline Baseline eGFR $\left(\mathrm{mL} / \mathrm{min} / 1.73 \mathrm{~m}^{2}\right)$ & $95.9 \pm 35.7$ & $98.6 \pm 33.4$ & $94.6 \pm 37.1$ & 0.04 \\
\hline Number of leads with STE & $4.2 \pm 1.5$ & $3.9 \pm 1.7$ & $4.7 \pm 2.0$ & 0.08 \\
\hline$>50 \%$ ST resolution at 90 minutes & $308(84.6 \%)$ & $276(87.1 \%)$ & $32(68.1 \%)$ & 0.03 \\
\hline LVEF at discharge $(\%)$ & $55.1 \pm 12.0$ & $56.8 \pm 11.9$ & $52.7 \pm 12.3$ & 0.02 \\
\hline
\end{tabular}

ACEI : Angiotensin converting enzyme inhibitor.

HbA1c. : Glycosylated hemoglobin.

ARB : Angiotensin receptor blocker.

CAD : Coronary artery disease.

eGFR : Estimated glomerular filtration rate.

HsTnT : High sensitive troponin T.

LVEF : Left ventricular ejection fraction.

STE : ST elevation.

Table (2): Comparison between procedural characteristics of both groups.

\begin{tabular}{|c|c|c|c|c|}
\hline & $\begin{array}{c}\text { Total } \\
(\mathrm{n}=364)\end{array}$ & $\begin{array}{l}\text { No MACE } \\
(\mathrm{n}=317)\end{array}$ & $\begin{array}{l}\text { MACE } \\
(\mathrm{n}=47)\end{array}$ & $\begin{array}{c}p- \\
\text { value }\end{array}$ \\
\hline Pain to balloon time (minutes) & $171.5 \pm 87.9$ & $150.3 \pm 74.8$ & $194.6 \pm 89.0$ & 0.01 \\
\hline Door to balloon (minutes) & $31.6 \pm 14.9$ & $30.2 \pm 13.5$ & $29.4 \pm 16.8$ & 0.15 \\
\hline TIMI Thrombus grade & $3.3 \pm 1.8$ & $3.3 \pm 1.4$ & $3.7 \pm 0.8$ & 0.02 \\
\hline Thrombus score & $5.8 \pm 3.7$ & $4.6 \pm 2.5$ & $6.9 \pm 2.7$ & $<0.001$ \\
\hline Proximal location of culprit lesion, $\mathrm{n}(\%)$ & $98(26.9 \%)$ & $76(24.0 \%)$ & $22(46.8 \%)$ & 0.001 \\
\hline Multivessel disease, $\mathrm{n}(\%)$ & $41(11.3 \%)$ & $35(11.0 \%)$ & $6(12.8 \%)$ & 0.06 \\
\hline IC Tirofiban administration, $\mathrm{n}(\%)$ & $171(47.0 \%)$ & $134(42.3 \%)$ & $37(78.7 \%)$ & 0.01 \\
\hline No of stents & $1.42 \pm 0.43$ & $1.40 \pm 0.45$ & $1.28 \pm 0.38$ & 0.52 \\
\hline Maximum inflation pressure (atm) & $14.1 \pm 2.1$ & $14.3 \pm 1.8$ & $13.9 \pm 2.8$ & 0.45 \\
\hline \multicolumn{5}{|l|}{ Postprocedural TIMI flow: } \\
\hline $0-1$ & $33(9.1 \%)$ & $17(5.4 \%)$ & $16(34.0 \%)$ & $<0.001$ \\
\hline $2-3$ & $331(90.9 \%)$ & $300(94.6 \%)$ & $31(66.0 \%)$ & $<0.001$ \\
\hline cTFC & $24.8 \pm 3.4$ & $22.1 \pm 4.3$ & $28.3 \pm 2.8$ & $<0.001$ \\
\hline \multicolumn{5}{|l|}{$M B G:$} \\
\hline $0-1$ & $42(11.5 \%)$ & $21(6.6 \%)$ & $21(44.7 \%)$ & $<0.001$ \\
\hline $2-3$ & $322(88.5 \%)$ & $296(93.4 \%)$ & $26(55.3 \%)$ & $<0.001$ \\
\hline No reflow, n (\%) & $17(4.7 \%)$ & $12(3.8 \%)$ & $5(10.6 \%)$ & $<0.001$ \\
\hline
\end{tabular}

cTFC: Corrected TIMI frame count. IC: Intracoronary. MBG: Myocardial blush grade. TIMI: Thrombolysis in myocardial infarction. 
Table (3): Independent predictors of MACE.

\begin{tabular}{|c|c|c|c|c|}
\hline & \multicolumn{2}{|c|}{ Univariate analysis } & \multicolumn{2}{|c|}{ Multivariate analysis } \\
\hline & $\mathrm{HR}(95 \% \mathrm{CI})$ & $p$ & $\operatorname{HR}(95 \% \mathrm{CI})$ & $p$ \\
\hline Female gender & $2.67(1.36-5.24)$ & 0.004 & $0.65(0.29-1.43)$ & 0.30 \\
\hline Current smoking & $2.14(1.1-4.1)$ & 0.024 & $1.43(0.66-3.144)$ & 0.36 \\
\hline Peak HsTnT & $1.04(1.0-1.09)$ & $<0.001$ & $1.02(0.96-1.06)$ & 0.31 \\
\hline Baseline eGFR & $1.75(1.13-2.77)$ & 0.003 & $1.52(0.93-2.41)$ & 0.087 \\
\hline LVEF at discharge & $1.26(0.92-1.56)$ & $<0.001$ & $1.18(1.06-1.63)$ & 0.01 \\
\hline Pain to balloon time (minutes) & $1.006(1.001-1.01)$ & 0.01 & $1.001(0.996-1.007)$ & 0.76 \\
\hline TIMI Thrombus grade & $1.32(0.99-1.75)$ & 0.05 & $0.79(0.51-1.23)$ & 0.30 \\
\hline Thrombus score & $1.42(1.23-1.64)$ & $<0.001$ & $1.43(1.19-1.73)$ & $<0.001$ \\
\hline Proximal location of culprit lesion & $1.22(0.94-1.73)$ & 0.04 & $1.14(0.92-1.75)$ & 0.16 \\
\hline IC Tirofiban administration & $0.99(0.99-1.00)$ & 0.014 & $0.99(0.99-1.00)$ & 0.093 \\
\hline No reflow & $1.07(1.03-1.11)$ & $<0.001$ & $1.04(0.98-1.10)$ & 0.082 \\
\hline
\end{tabular}

eGFR : Estimated glomerular filtration rate.

HsTnT : High sensitive troponin T.

LVEF: Left ventricular ejection fraction.

IC : Intracoronary.

TIMI : Thrombolysis in myocardial infarction.
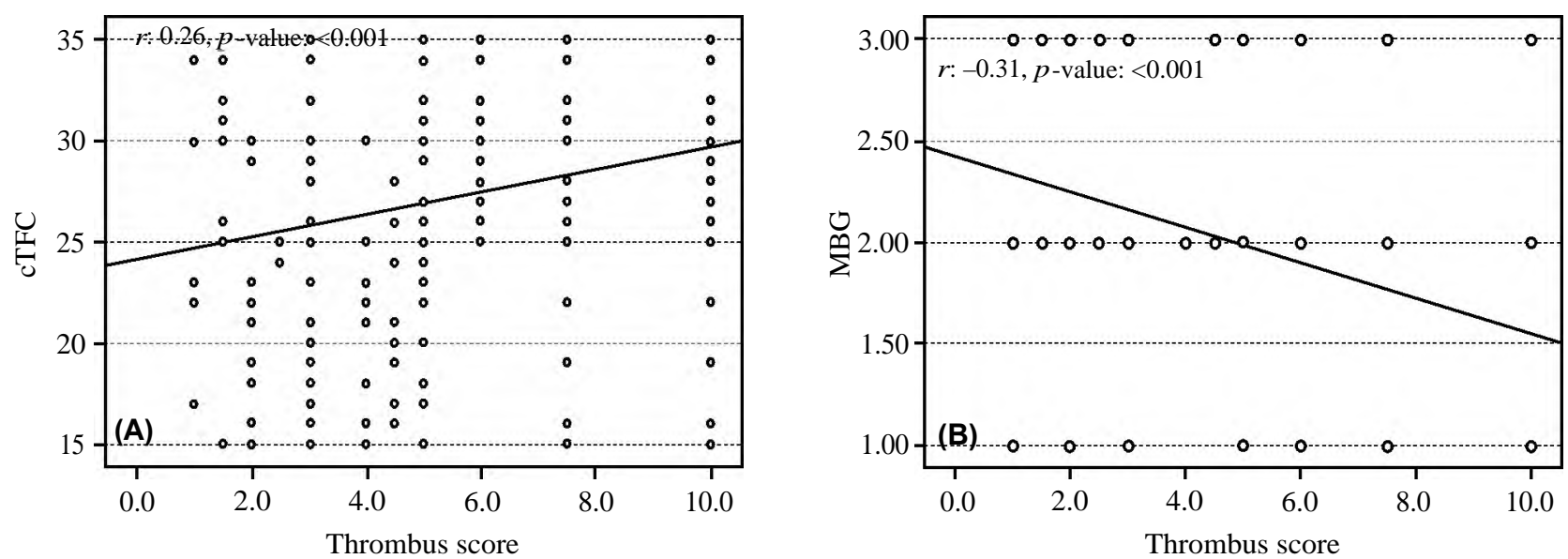

Fig. (2): Correlation between thrombus score and cTFC (A) and MBG (B).

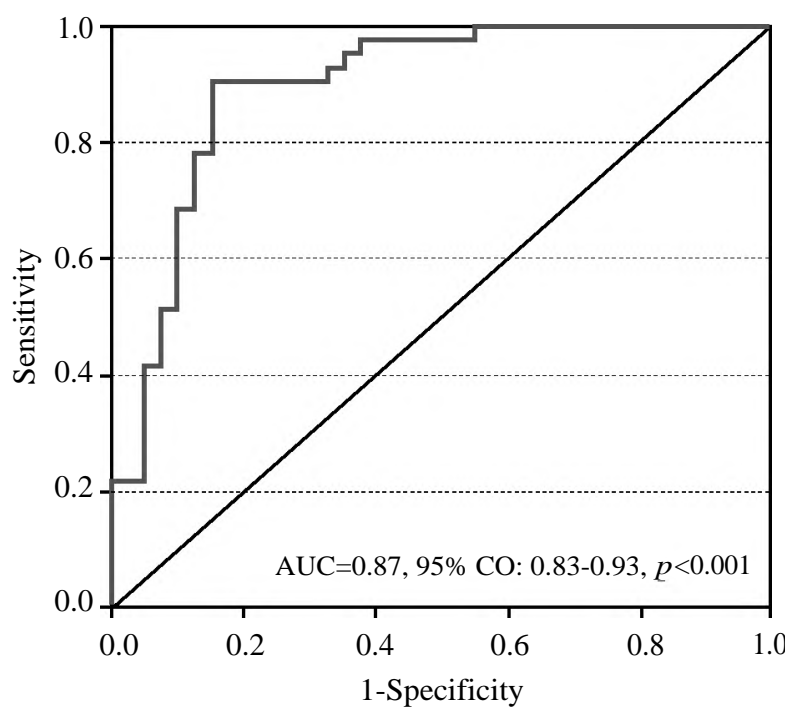

Fig. (3): ROC curve analysis to define the best cutoff value of thrombus score associated with MACE at 1 year.
Discussion

Predicting the risk of future MACE after STEMI has been, and remains, a subject of great interest. Early identification of patients at high risk of future MACE is essential to further optimize therapy and improve prognosis.

In the current study, we found that thrombus score, together with LVEF at hospital discharge, are independent predictors of MACE at 1 year after STEMI. Neither TIMI thrombus grade nor proximal lesion location could independently predict MACE. Despite data from thrombolytic era have suggested that proximal culprit lesion location was associated with increased mortality [23,24], this association was not found in the primary PCI era. In a primary PCI study, Noaman et al., [12] found that patients with proximal LAD culprits presented with higher 
rates of hemodynamic compromise and in-hospital and short-term MACE than patients with nonproximal LAD culprits. However, after multivariate adjustment, lesion location could not independently predict MACE. Consistent with these findings, we found that proximal lesion location couldn't independently predict MACE. Given these findings, weassume that the timeliness of mechanical reperfusion and the application of guideline-recommended adjunctive pharmacotherapy could attenuate the deleterious effect of proximal LAD artery culprits.

In the current study, we found that MACE was associated with larger thrombus burden, more use of IC tirofiban, and higher incidence of post-PCI TIMI 0-1 and no-reflow. The presence of large thrombi, per se, has been identified to be the main driving force for microvascular dysfunction downstream [25]. With the thrombus located proximally, the severity and extent of coronary microvascular dysfunction, and particularly microvascular occlusion (MVO), is expected to be greater. In our study, significant correlation was reported between thrombus score and indirect indicators of myocardial microvascular function (MBG and cTFC). Therefore, we assume that high thrombus score is associated with higher severity and extent of MVO.The latter has been previously proved by cardiac magnetic resonance (CMR) studies to be the strongest independent predictor of myocardium at risk, final infarct size and MACE [26]. This can provide supportour finding that thrombus score was the strongest independent predictor of MACE in our study. Of course, correlation of our novel scoring system with CMR indices is recommended for better understanding of this new score. If this correlation is proven, our angiographic score will have the relative advantages of simplicity, availability and applicability in the setting of primary PCI without extra cost.

Our study has some limitations: First, it was a single center study on a relatively small number of patients. Second, we intentionally excluded STEMI patients with non-thrombus containing culprits and those with cardiogenic shock because these patients have expected favorable and poor outcomes, respectively. Finally, the difference between outcomes of low TIMI thrombus grade in a proximal culprit as compared to high TIMI thrombus grade in a distal culprit (both would result in similar thrombus scores) was not studied.

\section{Conclusions:}

In STEMI patients treated by primary PCI; the novel angiographic "thrombus score", based on
TIMI thrombus grade and culprit lesion location, was the strongest independent predictor of MACE during the first year after STEMI. On the contrary, neither TIMI thrombus grade nor proximal culprit lesion location could independently predict MACE. Further studies are recommended to better understand and validate this new score.

Conflict of Interest: The authors report no conflicts of interest.

\section{References}

1- SZUMMER K., WALLENTIN L., LINDHAGEN L., et al.: Improved outcomes in patients with ST-elevation myocardial infarction during the last 20 years are related to implementation of evidence-based treatments: Experiences from the SWEDEHEART registry 1995-2014. Eur. Heart J., 38: 3056-3065, 2017.

2- ALI M., LANGE S.A., WITTLINGER T., et al.: In-hospital mortality after acute STEMI in patients undergoing primary PCI. Herz, 43 (8): 741-45, 2018.

3- HAUSENLOY D.J., BOTKER H.E., ENGSTROM T., et al.: Targeting reperfusion injury in patients with STsegment elevation myocardial infarction: Trials and tribulations. Eur. Heart J., 38: 935-941, 2017.

4- WU E., ORTIZ J.T., TEJEDOR P., et al.: Infarct size by contrast enhanced cardiac magnetic resonance is a stronger predictor of outcomes than left ventricular ejection fraction or end-systolic volume index: Prospective cohort study. Heart, 94: 730-736, 2008.

5- CHRISTIAN T., SCHWARTZ R. and GIBBONS R.: Determinants of infarct size in reperfusion therapy for acute myocardaial infarction. Circulation, 86: 81-90, 1992.

6- SIANOS G., PAPAFAKLIS M.I. and SERRUYS P.W.: Angiographic thrombus burden classification in patients with ST-segment elevation myocardial infarction treated with percutaneous coronary intervention. J. Invasive Cardiol., 22 (10 Suppl B): 6B-14B, 2010.

7- SINGH M., BERGER P.B., TING H.H., et al.: Influence of coronary thrombus on outcome of percutaneous coronary angioplasty in the current era (the Mayo Clinic experience). Am. J. Cardiol., 88 (10): 1091-1096, 2001.

8- HENRIQUES J.P., ZIJLSTRA F., OTTERVANGER J.P. et al.: Incidence and clinical significance of distal embolization during primary angioplasty for acute myocardial infarction. Eur. Heart J., 23: 1112-7, 2002.

9- TUNGSUBUTRA W., TOWASHIRAPORN K., TRESUKOSOL D., et al.: One-year clinical outcomes of ST segment elevation myocardial infarction patients treated with emergent percutaneous coronary intervention: The impact of thrombus burden. J. Med. Assoc. Thai., 97 (Suppl. 3): S139-S146, 2014.

10- TATLISU M.A., ADNAN K., MUHAMMED K., et al.: The association of the coronary thrombus burden with all-cause mortality and major cardiac events in segment elevation myocardial infarction patients treated with tirofiban. Coronary artery disease, 27 (7): 543-50, 2016. 
11- HARJAI K., MEHTA R.H., STONE G.W., et al.: Does proximal location of culprit lesion confer worse prognosis in patients undergoing primary percutaneous coronary intervention for ST elevation myocardial infarction? Journal of Interevntional Cardiology, 19 (4): 285-94, 2006.

12-NOAMAN S., GOH C.Y., VOGRIN S., et al.: Comparison of short-term clinical outcomes of proximal versus nonproximal lesion location in patients treated with primary percutaneous coronary intervention for ST-elevation myocardial infarction: The PROXIMITI study. Catheter Cardiovasc. Interv., 93: 32-40, 2019

13- TANG B and YANG H.: Post percutaneous coronary interventional outcomes on proximal vs non-proximal lesions of the left and right coronary arteries: A systematic review and meta-analysis. Medicine, 98: e16905, 2019.

14- GENSINI G.G.: A more meaningful scoring system for determining the severity of coronary heart disease. Am. J. Cardiol., 51: 606, 1983.

15- IBANEZ B., JAMES S., AGEWALL S., ANTUNES M.J., BUCCIARELLI-DUCCI C., BUENO H., et al.: 2017 ESC Guidelines for the management of acute myocardial infarction in patients presenting with ST-segment elevation: The Task Force for the management of acute myocardial infarction in patients presenting with ST-segment elevation of the European Society of Cardiology (ESC). Eur. Heart J., 39 (2): 119-177, 2018.

16- WILLIAMS B., MANCIA G., SPIERING W., AGABITI ROSEI E., AZIZI M., BURNIER M., et al.: 2018 ESC/ESH Guidelines for the managementof arterial hypertension: The Task Force for the management ofarterial hypertension of the European Society of Cardiology andthe European Society of Hypertension: The Task Force for the managementof arterial hypertension of the European Society ofCardiology and the European Society of Hypertension. J. Hypertens, 36 (10): 1953-2041, 2018.

17- American Diabetes Association: Standards of medical care for patientswith diabetes mellitus. Diabetes Care, 26 (Suppl 1): s33-50, 2003.

18- LEVEY A.S., BOSCH J.P., LEWIS J.B., GREENE T., ROGERS N. and ROTH D.: A more accurate method to estimate glomerular filtration rate from serum creatinine:
A new prediction equation. Modification of Diet in Renal Disease Study Group. Ann. Intern. Med., 130: 461-70, 1999.

19- GIBSON C.M., de LEMOS J.A., MURPHY S.A., et al.: Combination therapy with abciximab reduces angiographically evident thrombus in acute myocardial infarction: A TIMI 14 substudy. Circulation, 103: 2550-4, 2001.

20- The TIMI Study Group. Thrombolysis in myocardial infarction (TIMI) trial. N. Engl. J. Med., 312: 932-936, 1985.

21- GIBSON C.M., MURPHY S.A., RIZZO M.J., et al.: The relationship between the TIMI frame count and clinical outcomes after thrombolytic administration. Circulation, 99: 1945-50, 1999.

22- VAN'T HOF A.W.J., LIEM A., SURYAPRANATA H., et al.: Angiographic assessment of myocardial reperfusion in patients treated with primary angioplasty for acute myocardial infarction: Myocardial blush grade. Circulation, 97: 2302-6, 1998.

23- SCHUSTER E.H., GRIFFITH L.S. and BULKLEY B.H.: Preponderance of acute proximal left anterior descending coronary arterial lesions in fatal myocardial infarction: A clinicopathologic study. Am. J. Cardiol., 47: 1189-1196, 1981.

24- KARHA J., MURPHY S.A., KIRTANE A.J., de LEMOS J.A., AROESTY J.M., CANNON C.P., ANTMAN E.M., BRAUNWALD E., GIBSON C.M. and GROUP T.S.: Evaluation of the association of proximal coronary culprit artery lesion location with clinical outcomes in acute myocardial infarction. Am. J. Cardiology, 92: 913-918, 2003.

25- KIRMA C., IZGI A., DUNDAR C., et al.: Clinical and procedural predictors of no-reflow phenomenon after primary percutaneous coronary interventions. Circulation, 72: 716-721, 2008

26- WAHA S.D., DESCH S., EITEL I., et al.: Impact of early vs. late microvascular obstruction assessed by magnetic resonance imaging on long-term outcome after STelevation myocardial infarction: A comparison with traditional prognostic markers. Eur. Heart J., 31: 2660-2668, 2010. 


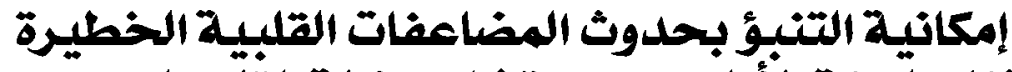

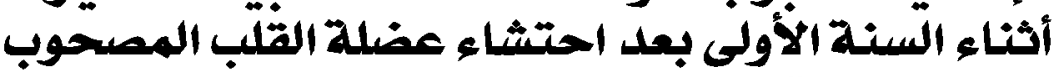

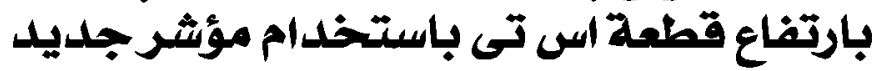

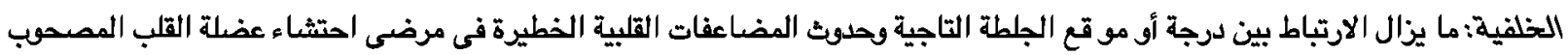

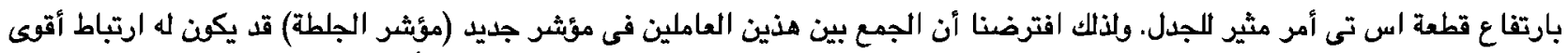

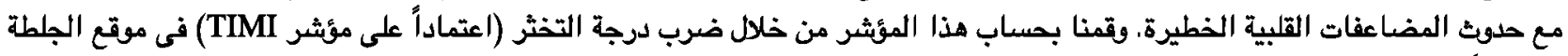
(اعتماداً على مؤشر المفيراث (Gensini)

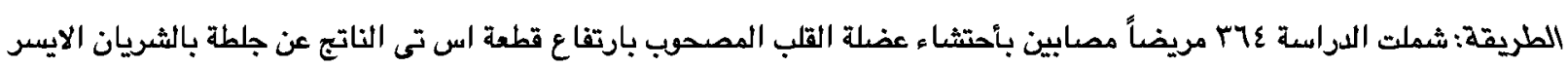

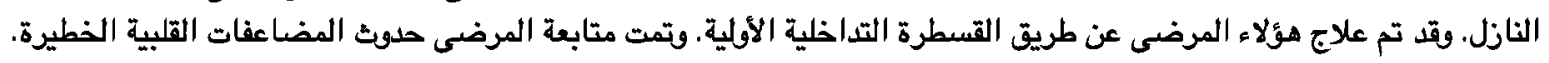

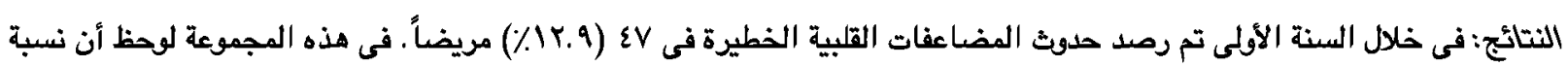

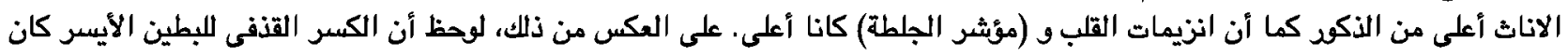

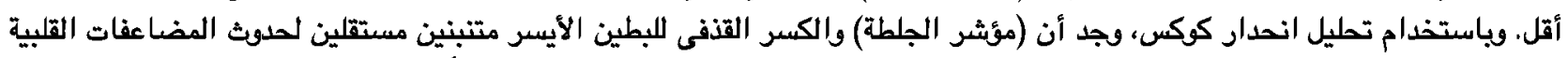

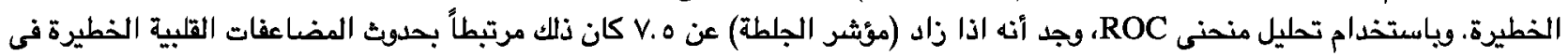

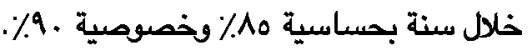
الخلاصة: فى مرضى احتشاء عضلة القلب الذين تم علاجهم بالقسطرة التداخلية الأولية كان (مؤشر الجلطة) الجديد أقوى متتبئ مستقل لحلوث المضاعفات القلبية الخطيرة خلال السنة الأولى. ولذلك نوصى بإجراء مزيد من الدراسات لفهم هذا المؤشر الجديد والتحقق منه بشكل 